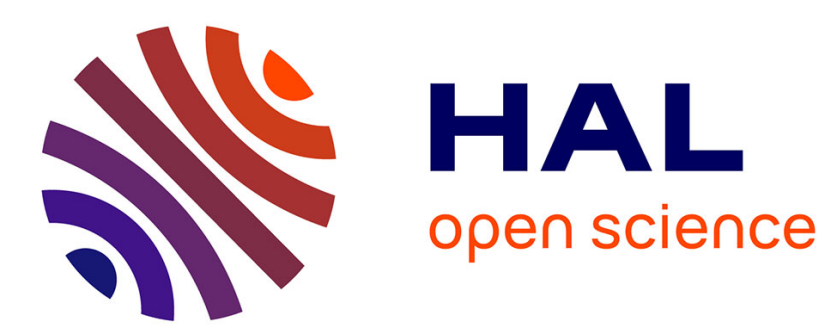

\title{
3D small strain large deflection beam shape sensing including poisson effect
}

Pierre-Loup Schaefer, Grégory Chagnon, Alexandre Moreau-Gaudry

\section{To cite this version:}

Pierre-Loup Schaefer, Grégory Chagnon, Alexandre Moreau-Gaudry. 3D small strain large deflection beam shape sensing including poisson effect. Engineering Structures, 2019, pp.109948. 10.1016/j.engstruct.2019.109948. hal-02425954

\section{HAL Id: hal-02425954 \\ https://hal.science/hal-02425954}

Submitted on 13 Mar 2020

HAL is a multi-disciplinary open access archive for the deposit and dissemination of scientific research documents, whether they are published or not. The documents may come from teaching and research institutions in France or abroad, or from public or private research centers.
L'archive ouverte pluridisciplinaire HAL, est destinée au dépôt et à la diffusion de documents scientifiques de niveau recherche, publiés ou non, émanant des établissements d'enseignement et de recherche français ou étrangers, des laboratoires publics ou privés. 


\title{
3D Small Strain Large Deflection Beam Shape Sensing Including Poisson Effect
}

\author{
Pierre-Loup Schaefer ${ }^{\mathrm{a}, *}$, Grégory Chagnon ${ }^{\mathrm{a}}$, Alexandre Moreau-Gaudry ${ }^{\mathrm{a}, \mathrm{b}}$ \\ ${ }^{a}$ TIMC-IMAG Laboratory, 5 Avenue du Grand Sablon, 38700 La Tronche, France \\ ${ }^{b}$ Grenoble Clinical Investigation Centre for Innovative Technology network, Pôle de santé publique, Département de \\ Méthodologie et de l'Information de Santé (DMIS), Institut de l'Ingénierie et de l'Information de Santé, CHU de Grenoble, \\ 38000 Grenoble, France.
}

\begin{abstract}
This article presents a new method to monitor beam shapes from strain measures. The beam is instrumented with strain sensors placed on its surface with an angle of orientation. The method developed is a 3D large deflection beam shape reconstruction based on a beam model incorporating Poisson effect and is thus able to take into account bending, torsion, shearing and tensile-compression deformations. Exemple of application on circular beams is conducted with beam shape reconstructed from FEM simulations strain measures. Results show that, compared to beam shape sensing methods using axial strain, the beam shape method proposed provides a significant increase in the reconstruction accuracy when the beam is subject to deformations containing torsion.
\end{abstract}

Keywords: Beam; Isotropic; Large deflection; Large displacement; Poisson effect; Rod; Shape sensing;

Small strain;

\section{Introduction}

Real-time shape monitoring of a beam structure is a problem with various domains of applications such as construction, medical field or aerospacial. An effective way to monitor a beam deformed shape consists in using cameras or optics which then provide directly the deformed beam shape. Nevertheless, this method can not be applicable everywhere due to lack of space or accessibility. In these cases the shape can be obtained from the measures of sensors implented in the structures, such as strain sensors. The beam shape is then not available directly and requires a reconstruction using the sensor data to be obtained. The topic of using strain sensors to monitor beam structures has been covered in the litterature with applications to the construction, medical and aeronautical field. In these works, the hypothesis concerning the beam

\footnotetext{
Whord count: 5098 words.

* Corresponding author

Email addresses: pierreloup.schaefer@gmail.com (Pierre-Loup Schaefer), gregory.chagnon@univ-grenoble-alpes.fr (Grégory Chagnon), alexandre.moreau-gaudry@univ-grenoble-alpes.fr (Alexandre Moreau-Gaudry)
} 


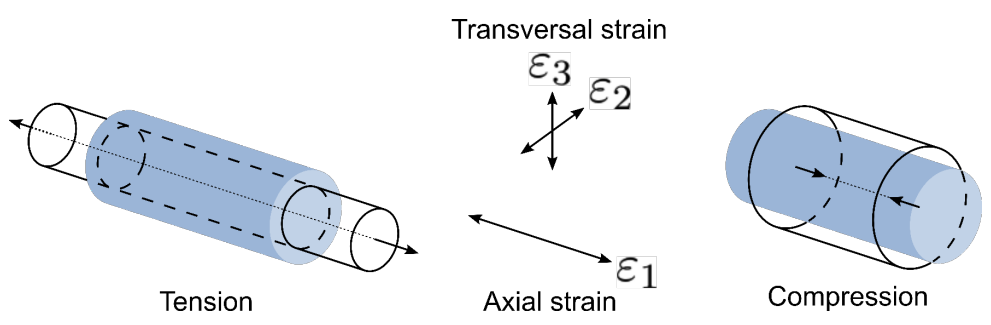

Figure 1: Poisson effect

deformation play a key role in the choice of the beam model used to reconstruct the shape. The modeling of the beam will thus notably be specific to the hypothesis concerning the cross-section of the beam during the deformation.

The Euler-Bernoulli beam theory makes the assumption the cross section remains rigid and perpendicular to the neutral axis. Different beam shape monitoring methods based on this hypothesis have been proposed over the years [42, 10, 13, 16, 22, 31, 45, 50, 36] with applications such as bridge monitoring [9, 11, 39, 47, minimally-invasive surgery [1, 33, 32, 38, 18, 26, 30, 43, 49, 37] or wing deformation [23]. In these methods, the bending informations of the beam are retrieved from the measures of the strain sensors placed parallel to the beam at its surface and are used to reconstruct the beam shape in $2 \mathrm{D}$ or $3 \mathrm{D}$. The torsion informations of the beam can be retrieved by placing the strain sensors not parallel to the beam such as in Askins et al. [3] and $\mathrm{Xu}$ et al. [46]. The deformation considered under the hypothesis of Euler-Bernoulli are bending, torsion and tensile-compression. The shearing deformation can be taken into account by assuming that the cross sections still remain rigid but are no longer perpendicular to the neutral axis, such as formulated in the Timoshenko beam theory. It is then possible to reconstruct the beam shape by taking into account this additional deformation type such as in Chadha et al. [6].

The validity of these hypothesis greatly depends of the characteristics of the beam, for as stated in Beck et al. [5], the choice of one hypothesis or the other involves generally the geometry of the beam. The hypothesis of Euler-Bernoulli will be used to model flexure-dominated ("slender") beam wheareas the Timoshenko theory will be prefered in a case of shear-dominated ("short") beams, these two theories still assuming that the cross-section of the beam remains rigid during the deformation. The validity of this last assumption also depends of the beam characteristics as some deformation of the cross section might occur, such as Poisson effect (in-plane deformation) and warping (out-of-plane deformation). The warping effect is influenced by the cross-section geometry, symmetry and beam thickness whereas the Poisson effect is a phenomenon caused during the deformation by the material characteristics. The work presented in this article is devoted to beam shape sensing including the in-plane deformation caused by the Poisson effect. As illustrated in Fig. 1 the Poisson effect appears during a material deformation and is characterized by the 
appearance of transversal strains $\varepsilon_{2}, \varepsilon_{3}$ caused by the axial strain $\varepsilon_{1}$ such that:

$$
\varepsilon_{i}=-\nu \varepsilon_{1}, \quad i=2,3
$$

where $\nu$ is the Poisson ratio of the material. Chadha et al. developed a beam shape sensing method including Poisson effect based on geometrically-exact beam theory containing a Poisson transformation intended to include the Poisson effect directly into the beam configuration [8, 7]. The beam configuration is noted $\xi_{1}, \xi_{2}, \xi_{3}$ in the reference state, with $\xi_{1}$ the position on the beam neutral axis and $\xi_{2}, \xi_{3}$ the position on the beam cross section. Due to the deformations caused by the Poisson effect, the position on the beam cross section at the current state are denoted $\hat{\xi}_{2}$ and $\hat{\xi}_{3}$. The axial strain is noted $\varepsilon_{1}$. The Poisson transformation defined by Chadha et al. is:

$$
\hat{\xi}_{i}=\left(1-\nu \varepsilon_{1}\left(\xi_{1}, \xi_{2}, \xi_{3}\right)\right) \xi_{i} \quad i=2,3
$$

By derivation of (2) one obtains:

$$
\frac{\partial \hat{\xi}_{i}}{\partial \xi_{i}}=1-\nu \varepsilon_{1}\left(\xi_{1}, \xi_{2}, \xi_{3}\right)-\nu \frac{\partial \varepsilon_{1}\left(\xi_{1}, \xi_{2}, \xi_{3}\right)}{\partial \xi_{i}} \quad i=2,3
$$

which can be rewritten as:

$$
\varepsilon_{i}=-\nu \varepsilon_{1}\left(\xi_{1}, \xi_{2}, \xi_{3}\right)-\nu \frac{\partial \varepsilon_{1}\left(\xi_{1}, \xi_{2}, \xi_{3}\right)}{\partial \xi_{i}} \quad i=2,3
$$

As a consequence, by using the Poisson transformation the transverse strain obtained in (4) thus differs from the transverse strain from Poisson relation (1) by an additional non-null term containing the derivative of $\varepsilon_{1}\left(\xi_{1}, \xi_{2}, \xi_{3}\right)$. In [7] the derivative $\partial \varepsilon_{1}\left(\xi_{1}, \xi_{2}, \xi_{3}\right) / \partial \xi_{i}$ is not null as it contains terms in $\xi_{2}$ and $\xi_{3}$ related to the bending deformation. The expression of the transverse strain obtained from the Poisson transformation thus differs from the Poisson relation. In [8] the axial strain $\varepsilon_{1}\left(\xi_{1}, \xi_{2}, \xi_{3}\right)$ is replaced by the midcurve axial strain $\left(\varepsilon_{1}\left(\xi_{1}, 0,0\right)=\varepsilon_{1}\left(\xi_{1}\right)\right)$ which leads to:

$$
\varepsilon_{i}=-\nu \varepsilon_{1}\left(\xi_{1}\right) \quad i=2,3
$$

Thus the transverse strain only depends of the midcurve axial strain and by consequences neglect the bending deformation. Consequently, the Poisson transformation is an approximation of the beam configuration which does not satisfy the Poisson relation.

Gherlone et al. [14, 15] has proposed a beam shape monitoring method including Poisson effect based on inverse finite element method. The method consists in reconstructing the three-dimensional displacement field of a beam structure from surfaced-measured strains by minimizing the square difference of the strain measures and the theoretical strains. The displacement field is then interpolated to give the full beam displacement. The configuration of beam being derived from the classical Timoshenko beam theory, the method is thus restricted to small rotations 24 . 


\section{Proposed approach}

We propose here a small strain large deflection beam shape sensing method taking into account the Poisson effect which allows to reconstruct the shapes of beams undergoing small strain deformations, with no restrictions on the amplitude of the deflections. Deflection refers to the movement of a beam from its original position due to the forces and loads being applied to the beam. In the following, the deflection of the beam denotes the distance related to the displacement of the free end of the beam between the reference and the deformed configurations. In the first part, the beam is first modeled using 3D beam theory and deformations in bending, torsion, shearing and tensile-compression are included in the configuration space. The Poisson phenomenon is then added in the expression of the small-strain strain tensor which is used to express the strain measure at the surface of the beam. The local strain measures at the surface are then used reversely to retrieve the local beam configuration. In the second part a reconstruction method is proposed to reconstruct the full shape of the beam undergoing large deflections and rotation deformations. An application on the circular beam is then presented and the impact of the Poisson effect on the strain parameters evaluations is evaluated. Finally, numerical simulations based on FEM were used to validate the reconstruction process and revelance of the strain model developed including the Poisson effect.

The beam model proposed is derived from the geometrically exact beam theory of Simo 40 from which is added the effect of the Poisson deformation directly in the small-strain tensor, thus providing a formulation of the beam strain in terms of bending, torsion, shearing, tensile/compression and Poisson ratio. By doing so it is then possible to choose the deformation to consider. The Poisson effect is implemented in the strain tensor and not in the configuration. In fact, the Poisson effect is moderate on the beam displacement but has huge impact on its deformation, which means that this effect impacts heavily the measures of the strain sensor but moderately their positions on the beam. The Poisson effect is thus only taken into account in the expression of the small-strain tensor and not in the beam configuration neither in the beam shape reconstruction as only the neutral axis of the beam is reconstructed and the Poisson effect has no impact on the neutral axis. This choice have double advantages as it allows to simplify the model and to inverse the relationships in order to express the beam configuration according to the strain measures. The necessity to take into account the Poisson effect and the validity of the strain model propose is demonstrated by comparison of beam shape reconstruction with FEM simulation of beam deformations. Moreover, the impact of the Poisson effect on the estimation of the strain configuration is expressed anatically. These validations shows that including

the Poisson effect in the strain tensor only allows to obtain accurate reconstruction. The difficulty of beam shape sensing lie in the fact that the model should be complete enough to be accurate but not too much to still be inversible, here being able to express beam configuration in terms of strains measures. To achieve this goal the model is restricted to small-strain and coupling effects are neglected. On the contrary of Gherlone, the strain measures are used to reconstruct the beam shape explicitly and by taking into account 


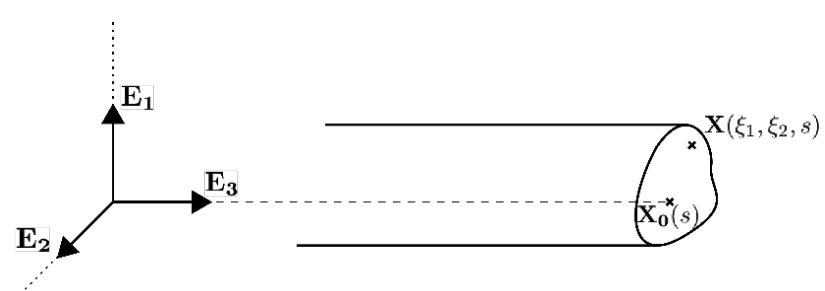

(a) Beam reference configuration

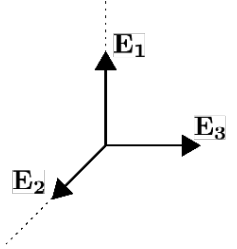

(b) Beam current configuration

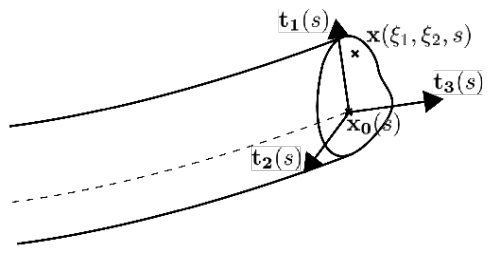

Figure 2: Beam different configurations

the large rotations and displacements deformations. To the best of author's knowledge, no beam shape method including Poisson effect and accounting for large rotations and displacements have been proposed before.

\section{Beam shape sensing including bending, torsion, shearing, tensile, compressive and Poisson deformations : General method}

This section presents the beam modeling, the strain tensor including Poisson effect, the expression of the beam configuration in terms of strain measures and the beam shape reconstruction method.

\subsection{Strain measure including Poisson effect for isotropic beams}

The beam is modeled using the beam theory developed by Simo [40] under the hypothesis of linear elasticity and its material is supposed isotropic. The beam is straight at the reference configuration. The reference coordinates is the orthonormal frame $\left(\mathbf{E}_{\mathbf{1}}, \mathbf{E}_{\mathbf{2}}, \mathbf{E}_{\mathbf{3}}\right)$. The position on the neutral axis at length $s$ is noted $\mathbf{X}_{\mathbf{0}}(s)$ in the reference configuration and $\mathbf{x}_{\mathbf{0}}(s)$ in the current configuration. The position of a point of the beam located at length $s$ along $\mathbf{E}_{\mathbf{3}}$ and radial position $\xi_{1}$ and $\xi_{2}$ along $\mathbf{E}_{\mathbf{1}}$ and $\mathbf{E}_{\mathbf{2}}$ is noted $\mathbf{X}(\boldsymbol{\xi}, s)$ in the reference configuration and $\mathbf{x}(\boldsymbol{\xi}, s)$ in the current configuration with $\boldsymbol{\xi}=\xi_{1} \mathbf{E}_{\mathbf{1}}+\xi_{2} \mathbf{E}_{\mathbf{2}}$. At the reference configuration we then have:

$$
\mathbf{X}(\boldsymbol{\xi}, s)=\xi_{1} \mathbf{E}_{\mathbf{1}}+\xi_{2} \mathbf{E}_{\mathbf{2}}+s \mathbf{E}_{\mathbf{3}}
$$

The orthonormal moving frame $\left(\mathbf{t}_{\mathbf{1}}(s), \mathbf{t}_{\mathbf{2}}(s), \mathbf{t}_{\mathbf{3}}(s)\right)$ is defined such that the current configuration $\mathbf{x}(\boldsymbol{\xi}, s)$ of the beam is noted:

$$
\mathbf{x}(\boldsymbol{\xi}, s)=\xi_{1} \mathbf{t}_{\mathbf{1}}(s)+\xi_{2} \mathbf{t}_{\mathbf{2}}(s)+\mathbf{x}_{\mathbf{0}}(s)
$$

Reference and current beam configurations are illustrated respectively in Fig. 2a and Fig. 2b. As the moving frame $\left(\mathbf{t}_{\mathbf{1}}(s), \mathbf{t}_{\mathbf{2}}(s), \mathbf{t}_{\mathbf{3}}(s)\right)$ is orthonormal for each $s$, there exists an orthonormal transformation $s \rightarrow \boldsymbol{\Lambda}(s) \in S O(3)$ such that:

$$
\forall I \in \llbracket 1,3 \rrbracket, \mathbf{t}_{\mathbf{I}}(s)=\boldsymbol{\Lambda}(s) \mathbf{E}_{\mathbf{I}}, \quad \boldsymbol{\Lambda}(s)=\Lambda_{i, j}(s) \mathbf{E}_{\mathbf{i}} \otimes \mathbf{E}_{\mathbf{j}}
$$




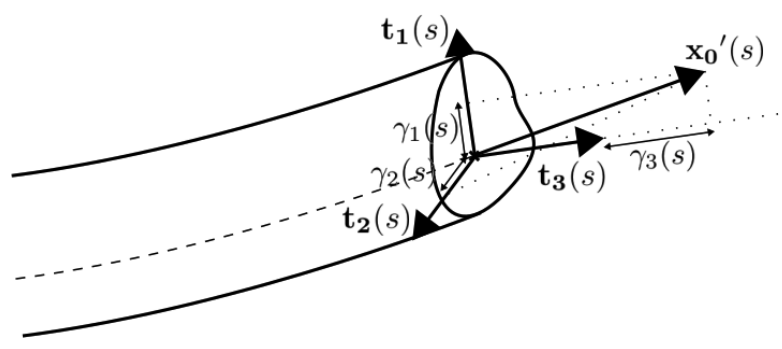

Figure 3: Beam shearing coefficients

The derivative of the frame $\left(\mathbf{t}_{\mathbf{1}}, \mathbf{t}_{\mathbf{2}}, \mathbf{t}_{\mathbf{3}}\right)$ according to $s$ can be written $\mathbf{t}_{\mathbf{i}}{ }^{\prime}(s)=\boldsymbol{\Omega}(s) \mathbf{t}_{\mathbf{i}}(s)$ where $\boldsymbol{\Omega}$ is a skew-symmetric tensor defined by:

$$
\boldsymbol{\Omega}(s)=\boldsymbol{\Lambda}^{\prime}(s) \boldsymbol{\Lambda}^{T}(s)
$$

The tensor $\boldsymbol{\Omega}$ can be expressed relatively to the moving frame $\left(\mathbf{t}_{\mathbf{1}}, \mathbf{t}_{\mathbf{2}}, \mathbf{t}_{\mathbf{3}}\right)$ with the coefficients $\kappa_{1}, \kappa_{2}, \kappa_{3}$. The expression of $\boldsymbol{\Omega}$ in the basis $\left(\mathbf{t}_{\mathbf{1}}, \mathbf{t}_{\mathbf{2}}, \mathbf{t}_{\mathbf{3}}\right)$ is then:

$$
[\boldsymbol{\Omega}(s)]_{\mathbf{t}}=\left[\begin{array}{ccc}
0 & -\kappa_{3}(s) & \kappa_{2}(s) \\
\kappa_{3}(s) & 0 & -\kappa_{1}(s) \\
-\kappa_{2}(s) & \kappa_{1}(s) & 0
\end{array}\right]
$$

The coordinates of $\mathbf{x}_{\mathbf{0}}{ }^{\prime}$ in the frame $\left(\mathbf{t}_{\mathbf{1}}, \mathbf{t}_{\mathbf{2}}, \mathbf{t}_{\mathbf{3}}\right)$ are denoted $\gamma_{1}, \gamma_{2}$ and $1+\gamma_{3}$ :

$$
\mathbf{x}_{\mathbf{0}}{ }^{\prime}(s)=\gamma_{1}(s) \mathbf{t}_{\mathbf{1}}(s)+\gamma_{2}(s) \mathbf{t}_{\mathbf{2}}(s)+\left(1+\gamma_{3}(s)\right) \mathbf{t}_{\mathbf{3}}(s)
$$

The parameters $\kappa_{i}$ and $\gamma_{i}$ are respectively the rotational and translational strain measures conjugate to the stress acting on the cross sections. More precisely, the components $\kappa_{1}$ and $\kappa_{2}$ are the bending strains whereas $\kappa_{3}$ is the twist rate change. The components $\gamma_{1}$ and $\gamma_{2}$ are the shear strains and $\gamma_{3}$ is the extensional strain [12, 20]. The parameters $\kappa_{i}$ and $\gamma_{i}$ define the beam configuration. The deformation gradient $\mathbf{F}$ is a two-point tensor containing the derivatives of the current configuration according to the reference configuration:

$$
\begin{aligned}
\mathbf{F} & =\frac{\partial \mathbf{x}}{\partial \mathbf{X}} \\
& =\frac{\partial \mathbf{x}}{\partial X_{j}} \otimes \mathbf{E}_{\mathbf{j}}
\end{aligned}
$$

Using the reference beam configuration in (6) we have:

$$
\mathbf{F}=\frac{\partial \mathbf{x}}{\partial \xi_{1}} \otimes \mathbf{E}_{\mathbf{1}}+\frac{\partial \mathbf{x}}{\partial \xi_{2}} \otimes \mathbf{E}_{\mathbf{2}}+\frac{\partial \mathbf{x}}{\partial s} \otimes \mathbf{E}_{\mathbf{3}}
$$

And from (7) we have:

$$
\frac{\partial \mathbf{x}}{\partial \xi_{1}}=\mathbf{t}_{\mathbf{1}}, \quad \frac{\partial \mathbf{x}}{\partial \xi_{2}}=\mathbf{t}_{\mathbf{2}}, \quad \frac{\partial \mathbf{x}}{\partial s}=\xi_{1} \mathbf{t}_{\mathbf{1}}{ }^{\prime}+\xi_{2} \mathbf{t}_{\mathbf{2}}{ }^{\prime}+\mathbf{x}_{\mathbf{0}}{ }^{\prime}
$$


Consequently:

$$
\begin{aligned}
\mathbf{F} & =\mathbf{t}_{\mathbf{1}} \otimes \mathbf{E}_{\mathbf{1}}+\mathbf{t}_{\mathbf{2}} \otimes \mathbf{E}_{\mathbf{2}}+\left(\xi_{1} \mathbf{t}_{\mathbf{1}}{ }^{\prime}+\xi_{2} \mathbf{t}_{\mathbf{2}}{ }^{\prime}+\mathbf{x}_{\mathbf{0}}{ }^{\prime}\right) \otimes \mathbf{E}_{\mathbf{3}} \\
& =\mathbf{t}_{\mathbf{i}} \otimes \mathbf{E}_{\mathbf{i}}+\left(\xi_{1} \mathbf{t}_{\mathbf{1}}{ }^{\prime}+\xi_{2} \mathbf{t}_{\mathbf{2}}{ }^{\prime}+\mathbf{x}_{\mathbf{0}}{ }^{\prime}-\mathbf{t}_{\mathbf{3}}\right) \otimes \mathbf{E}_{\mathbf{3}} \\
& =\boldsymbol{\Lambda}+\mathbf{e} \otimes \mathbf{E}_{\mathbf{3}}
\end{aligned}
$$

with:

$$
\mathbf{e}=\xi_{1} \mathbf{t}_{\mathbf{1}}{ }^{\prime}+\xi_{2} \mathbf{t}_{\mathbf{2}}{ }^{\prime}+\mathbf{x}_{\mathbf{0}}{ }^{\prime}-\mathbf{t}_{\mathbf{3}}
$$

which gives:

$$
\mathbf{e}=\left(\gamma_{1}-\xi_{2} \kappa_{3}\right) \mathbf{t}_{\mathbf{1}}+\left(\gamma_{2}+\xi_{1} \kappa_{3}\right) \mathbf{t}_{\mathbf{2}}+\left(\gamma_{3}+\xi_{2} \kappa_{1}-\xi_{1} \kappa_{2}\right) \mathbf{t}_{\mathbf{3}}
$$

Thus $\mathbf{F}$ is the sum of the rotation tensor $\boldsymbol{\Lambda}$ and the tensor $\mathbf{e} \otimes \mathbf{E}_{\mathbf{3}}$ containing the pure current strain vector $\mathbf{e}$ 35. It should be noted that for any vector $\boldsymbol{\xi}=\xi_{1} \mathbf{E}_{\mathbf{1}}+\xi_{2} \mathbf{E}_{\mathbf{2}}$ included in the cross-section we have $\mathbf{F} \boldsymbol{\xi}=\boldsymbol{\Lambda} \boldsymbol{\xi}$ which a rotation of that vector. This illustrates the rigidity hypothesis of the cross section during the beam deformation. The definition of the Green-Lagrange strain tensor is:

$$
\mathbf{E}=\frac{1}{2}\left(\mathbf{F}^{T} \mathbf{F}-\mathbf{I}\right)
$$

Inserting the expression of $\mathbf{F}$ from 180 in 21 gives:

$$
\mathbf{E}=\frac{1}{2}\left(\left(\boldsymbol{\Lambda}+\mathbf{e} \otimes \mathbf{E}_{\mathbf{3}}\right)^{T}\left(\boldsymbol{\Lambda}+\mathbf{e} \otimes \mathbf{E}_{\mathbf{3}}\right)-\mathbf{I}\right)
$$

and using the expression of $\mathbf{e}$ from 20 thus provides the following expression of the Green-Lagrange strain tensor $\mathbf{E}$ :

$$
\mathbf{E}=\frac{1}{2}\left(\left(\mathbf{e . t} \mathbf{t}_{\mathbf{i}}\right)\left(\mathbf{E}_{\mathbf{i}} \otimes \mathbf{E}_{\mathbf{3}}+\mathbf{E}_{\mathbf{3}} \otimes \mathbf{E}_{\mathbf{i}}\right)+(\text { e.e }) \mathbf{E}_{\mathbf{3}} \otimes \mathbf{E}_{\mathbf{3}}\right)
$$

The small-strain tensor $\varepsilon$ is then obtained by neglecting the quadratic part of the expression of the finite strain tensor 23 :

$$
\varepsilon=\frac{1}{2}\left(\left(\mathbf{e . t} \mathbf{t}_{\mathbf{i}}\right)\left(\mathbf{E}_{\mathbf{i}} \otimes \mathbf{E}_{\mathbf{3}}+\mathbf{E}_{\mathbf{3}} \otimes \mathbf{E}_{\mathbf{i}}\right)\right)
$$

The expression of the small strain tensor $\varepsilon$ in the basis $\mathbf{E}_{\mathbf{i}} \otimes \mathbf{E}_{\mathbf{j}}$ is thus:

$$
\varepsilon=\frac{1}{2}\left[\begin{array}{ccc}
0 & 0 & \text { e.t } \\
0 & 0 & \text { e.t } \\
\text { e.t } & \text { e.t } & 2 \text { e.t } \\
\mathbf{t}_{3}
\end{array}\right]
$$

This beam model does not yet take into account the Poisson effect. The transverse strains created by this effect from the normal strains are thus ignored, which deteriorate the strain accuracy given by the model. Rather than trying to incorporate it into the beam configuration, the Poisson effect will be added into the small strain tensor formulation 24] following a method used by Weiss [44, Linn et al. 25] and Liu et al. 
27. As shown in 25. the coefficients $\varepsilon_{11}, \varepsilon_{12}, \varepsilon_{21}, \varepsilon_{22}$ of the tensor $\varepsilon$ are null due to the rigid cross section assumption which restricts the inclusion of Poisson's effect [7. The idea consists to allow uniform lateral contraction of the cross section by including the transverse strains due to the normal strain orthogonal to the cross section. The small-strain tensor $\tilde{\varepsilon}$ taking into account the Poisson effect is then defined the following way 25, 27]:

$$
\tilde{\varepsilon}_{i j}=\left\{\begin{array}{rr}
-\nu \varepsilon_{33}, & (i, j) \in\{(1,1),(2,2)\} \\
\varepsilon_{i j}, & (i, j) \notin\{(1,1),(2,2)\}
\end{array}\right.
$$

According to the expression of $\varepsilon$ in (24) we then have:

$$
\begin{aligned}
\tilde{\varepsilon}= & \frac{1}{2}\left(\mathbf{e . t} \mathbf{t}_{\mathbf{i}}\right)\left(\mathbf{E}_{\mathbf{i}} \otimes \mathbf{E}_{\mathbf{3}}+\mathbf{E}_{\mathbf{3}} \otimes \mathbf{E}_{\mathbf{i}}\right) \\
& -\nu \mathbf{e . t _ { 3 }}\left(\mathbf{E}_{\mathbf{1}} \otimes \mathbf{E}_{\mathbf{1}}+\mathbf{E}_{\mathbf{2}} \otimes \mathbf{E}_{\mathbf{2}}\right)
\end{aligned}
$$

And the expression of the strain tensor $\tilde{\varepsilon}$ in the basis $\mathbf{E}_{\mathbf{i}} \otimes \mathbf{E}_{\mathbf{j}}$ is then:

$$
\tilde{\varepsilon}=\frac{1}{2}\left[\begin{array}{ccc}
-2 \nu \text { e.t } & 0 & \text { e.t } \\
0 & -2 \nu \text { e.t } & \text { e.t } \\
\text { e.t } & \text { e.t }_{\mathbf{1}} & 2 \text { e. } \mathbf{t}_{3}
\end{array}\right]
$$

\subsection{Objectivity of the strain measure}

In order to have a method taking into account large displacement and rotation it is necessary to check that the strain measure used is objective [35]. The objectivity of material strain measure at a particular configuration is understood as their inherent ability to remain unaffected by a constant motion of the configuration [12. In order to prove the objectivity of the small-strain tensor including Poisson deformation proposed in 27) we will demonstrate the invariance of its expression under a rigid body motion decomposed in translation $\mathbf{c} \in \mathbb{R}^{3}$ and rotation $\mathbf{Q} \in S O(3)$. This rigid body motion modifies the current configuration of the beam such that:

$$
\begin{aligned}
& \mathbf{x}_{\mathbf{0}}^{*}=\mathbf{c}+\mathbf{Q x _ { 0 }} \\
& \mathbf{t}_{\mathbf{i}}^{*}=\mathbf{Q t}_{\mathbf{i}}
\end{aligned}
$$


The strain vector and the small strain tensor undergoing the rigid body motion are denoted $\mathbf{e}^{*}$ and $\tilde{\varepsilon}^{*}$. According to 20 the expression of the strain vector $\mathbf{e}^{*}$ is then:

$$
\begin{aligned}
\mathbf{e}^{*}= & \xi_{1} \mathbf{t}_{\mathbf{1}}^{* \prime}+\xi_{2} \mathbf{t}_{\mathbf{2}}^{* \prime}+\mathbf{x}_{\mathbf{0}}^{* \prime}-\mathbf{t}_{\mathbf{3}}^{*} \\
= & \xi_{1}\left(\mathbf{Q} \mathbf{t}_{\mathbf{1}}\right)^{\prime}+\xi_{2}\left(\mathbf{Q \mathbf { t } _ { 2 }}\right)^{\prime}+\left(\mathbf{c}+\mathbf{Q x _ { \mathbf { 0 } }}\right)^{\prime}-\mathbf{Q} \mathbf{t}_{\mathbf{3}} \\
= & \xi_{1}\left(\mathbf{Q}^{\prime} \mathbf{t}_{\mathbf{1}}+\mathbf{Q t}_{\mathbf{1}}{ }^{\prime}\right)+\xi_{2}\left(\mathbf{Q}^{\prime} \mathbf{t}_{\mathbf{2}}+\mathbf{Q} \mathbf{t}_{\mathbf{2}}{ }^{\prime}\right) \\
& +\left(\mathbf{c}^{\prime}+\mathbf{Q}^{\prime} \mathbf{x}_{\mathbf{0}}+\mathbf{Q} \mathbf{x}_{\mathbf{0}}{ }^{\prime}\right)-\mathbf{Q} \mathbf{t}_{\mathbf{3}} \\
= & \xi_{1} \mathbf{Q} \mathbf{t}_{\mathbf{1}}{ }^{\prime}+\xi_{2} \mathbf{Q} \mathbf{t}_{\mathbf{2}}{ }^{\prime}+\mathbf{Q} \mathbf{x}_{\mathbf{0}}{ }^{\prime}-\mathbf{Q} \mathbf{t}_{\mathbf{3}} \\
= & \mathbf{Q}\left(\xi_{1} \mathbf{t}_{\mathbf{1}}{ }^{\prime}+\xi_{2} \mathbf{t}_{\mathbf{2}}{ }^{\prime}+\mathbf{x}_{\mathbf{0}}{ }^{\prime}-\mathbf{t}_{\mathbf{3}}\right) \\
= & \mathbf{Q e}
\end{aligned}
$$

As the scalar product remains invariant by orthogonal transformations:

$$
\begin{aligned}
\mathbf{e}^{*} \cdot \mathbf{t}_{\mathbf{i}}^{*} & =\mathbf{Q e} \cdot \mathbf{Q} \mathbf{t}_{\mathbf{i}} \\
& =\mathbf{e} \cdot \mathbf{t}_{\mathbf{i}}
\end{aligned}
$$

Consequently, according to the tensor definition in 27) we then have:

$$
\tilde{\varepsilon}^{*}=\tilde{\varepsilon}
$$

Finally, the small-strain tensor including Poisson effect $\tilde{\varepsilon}$ is invariant under rigid body motions and is then an objective strain measure.

\subsection{Beam configuration from strain measures}

Let $\mathbf{U}=U_{i} \mathbf{E}_{\mathbf{i}}$ be a unit vector in the reference undeformed configuration noted $\mathbf{u}$ in the deformed current configuration. The normal strain including Poisson effect at location $(\boldsymbol{\xi}, s)$ in the direction $\mathbf{U}$ for a material with Poisson ratio $\nu$ is noted $\varepsilon$ and can then be expressed using the tensor $\tilde{\varepsilon}$ :

$$
\varepsilon=\mathbf{U} \tilde{\varepsilon}(\boldsymbol{\xi}, s, \nu) \mathbf{U}
$$

Using the expression of $\tilde{\varepsilon}$ in 27 then gives:

$$
\varepsilon=\mathbf{e}(\boldsymbol{\xi}, s) \cdot \mathbf{t}_{\mathbf{i}}(s) U_{i} U_{3}-\nu \mathbf{e}(\boldsymbol{\xi}, s) \cdot \mathbf{t}_{\mathbf{3}}(s)\left(U_{1}^{2}+U_{2}^{2}\right)
$$

The expression 42 shows that the inclusion of the Poisson effect in our model leads to the appearance of the new term $\nu \mathbf{e}(\boldsymbol{\xi}, s) \cdot \mathbf{t}_{\mathbf{3}}(s)\left(U_{1}^{2}+U_{2}^{2}\right)$ in the strain expression. From the definition of the strain vector in 20 results the following scalar relations:

$$
\begin{aligned}
& \mathbf{e}(\boldsymbol{\xi}, s) \cdot \mathbf{t}_{\mathbf{1}}(s)=\gamma_{1}(s)-\xi_{2} \kappa_{3}(s) \\
& \mathbf{e}(\boldsymbol{\xi}, s) \cdot \mathbf{t}_{\mathbf{2}}(s)=\gamma_{2}(s)+\xi_{1} \kappa_{3}(s) \\
& \mathbf{e}(\boldsymbol{\xi}, s) \cdot \mathbf{t}_{\mathbf{3}}(s)=\gamma_{3}(s)+\xi_{2} \kappa_{1}(s)-\xi_{1} \kappa_{2}(s)
\end{aligned}
$$


Using the relations (43), 444 and (45) the strain $\varepsilon$ can therefore be expressed as the scalar product between the beam position and direction vector $\mathbf{V}(\boldsymbol{\xi}, \mathbf{U}, \nu)$ and the beam configuration vector $\mathbf{K}(s)$ :

$$
\varepsilon=\mathbf{V}(\boldsymbol{\xi}, \mathbf{U}, \nu) \cdot \mathbf{K}(s)
$$

with the vector field $\mathbf{V}: \mathbb{R}^{2} \times \mathbb{R}^{3} \times \mathbb{R} \rightarrow \mathbb{R}^{6}$ having the following components $V_{i}$ :

$$
\begin{aligned}
& V_{1}(\boldsymbol{\xi}, \mathbf{U}, \nu)=\xi_{2}\left(U_{3}^{2}-\nu\left(U_{1}^{2}+U_{2}^{2}\right)\right) \\
& V_{2}(\boldsymbol{\xi}, \mathbf{U}, \nu)=-\xi_{1}\left(U_{3}^{2}-\nu\left(U_{1}^{2}+U_{2}^{2}\right)\right) \\
& V_{3}(\boldsymbol{\xi}, \mathbf{U}, \nu)=U_{3}\left(\xi_{1} U_{2}-\xi_{2} U_{1}\right) \\
& V_{4}(\boldsymbol{\xi}, \mathbf{U}, \nu)=U_{1} U_{3} \\
& V_{5}(\boldsymbol{\xi}, \mathbf{U}, \nu)=U_{2} U_{3} \\
& V_{6}(\boldsymbol{\xi}, \mathbf{U}, \nu)=U_{3}^{2}-\nu\left(U_{1}^{2}+U_{2}^{2}\right)
\end{aligned}
$$

and the vector field $\mathbf{K}: \mathbb{R} \rightarrow \mathbb{R}^{6}$ defined by:

$$
\begin{aligned}
& K_{1}(s)=\kappa_{1}(s) \\
& K_{2}(s)=\kappa_{2}(s) \\
& K_{3}(s)=\kappa_{3}(s) \\
& K_{4}(s)=\gamma_{1}(s) \\
& K_{5}(s)=\gamma_{2}(s) \\
& K_{6}(s)=\gamma_{3}(s)
\end{aligned}
$$

The strain measured at location $\left(\boldsymbol{\xi}_{\boldsymbol{k}}, s\right)$ in the direction $\mathbf{U}_{\mathbf{k}}$ is noted $\varepsilon_{k}$ with $k \in I$. As six strain measures are necessary to retrieve the full strain configuration of a beam cross section, we pose $I=\llbracket 1,6 \rrbracket$. The vector composed of the strain measures is noted $\mathbf{B}$ with $B_{i}=\varepsilon_{i}$. Using (46) the system associating the strain configuration $\mathbf{K}(s)$ to the strain measures $\mathbf{B}$ is defined by:

$$
\mathbf{A}\left(\boldsymbol{\xi}_{\boldsymbol{k}}, \mathbf{U}_{\mathbf{k}}, \nu\right) \mathbf{K}(s)=\mathbf{B}
$$

with $\mathbf{A}$ the matrix of the system such that:

$$
A_{i, j}=V_{j}\left(\boldsymbol{\xi}_{i}, \mathbf{U}_{\mathbf{i}}, \nu\right)
$$

Under the assumption of the inversibility of matrix $\mathbf{A}$, the beam configuration of a cross section $\mathbf{K}(s)$ can then be expressed from the strain measures $\mathbf{B}$ on that cross section:

$$
\mathbf{K}(s)=\mathbf{A}^{-\mathbf{1}}\left(\boldsymbol{\xi}_{\boldsymbol{k}}, \mathbf{U}_{\mathbf{k}}, \nu\right) \mathbf{B}
$$




\subsection{Beam shape reconstruction}

The resolution of system (59) gives the values of each parameters $\kappa_{i}(s)$ and $\gamma_{i}(s)$ at each abscissa $s$ where strain measures are made. The interpolation of those values gives the estimates of $\kappa_{i}$ and $\gamma_{i}$ on the whole length of the beam. From (9) we have the following relation where $[\cdot]_{\mathbf{E}}$ is the matrix form of a tensor in the $\left(\mathbf{E}_{1}, \mathbf{E}_{\mathbf{2}}, \mathbf{E}_{\mathbf{3}}\right)$ basis:

$$
\left[\boldsymbol{\Lambda}^{\prime}\right]_{\mathbf{E}}=[\boldsymbol{\Omega}]_{\mathbf{E}}[\boldsymbol{\Lambda}]_{\mathbf{E}}
$$

Using the transformation matrix $[\boldsymbol{\Lambda}]$ we have by coordinate transformations of tensor components:

$$
[\boldsymbol{\Omega}]_{\mathbf{E}}=[\boldsymbol{\Lambda}][\boldsymbol{\Omega}]_{\mathbf{t}}\left[\boldsymbol{\Lambda}^{T}\right]
$$

Finally using the fact that the matrix form of the tensor $\Lambda$ in the basis $\left(\mathbf{E}_{\mathbf{1}}, \mathbf{E}_{\mathbf{2}}, \mathbf{E}_{\mathbf{3}}\right)$ is equal to the transformation matrix $[\boldsymbol{\Lambda}]$ we have:

$$
\left[\boldsymbol{\Lambda}^{T}\right]_{\mathbf{E}}^{\prime}=\left[\boldsymbol{\Omega}^{T}\right]_{\mathbf{t}}\left[\boldsymbol{\Lambda}^{T}\right]_{\mathbf{E}}
$$

Let $t_{i}^{j}$ be the components of vector $\mathbf{t}_{\mathbf{i}}$ in the basis $\left(\mathbf{E}_{\mathbf{1}}, \mathbf{E}_{\mathbf{2}}, \mathbf{E}_{\mathbf{3}}\right)$ such that $\mathbf{t}_{\mathbf{i}}=t_{i}^{j} \mathbf{E}_{\mathbf{j}}$. From 64 we build the following matrix differential equation:

$$
\begin{aligned}
\mathbf{Y}^{\prime} & =\mathbf{A Y} \\
\mathbf{Y}(0) & =\mathbf{Y}_{\mathbf{0}}
\end{aligned}
$$

with:

$$
\mathbf{Y}=\left[\begin{array}{ccc}
t_{1}^{1} & t_{1}^{2} & t_{1}^{3} \\
t_{2}^{1} & t_{2}^{2} & t_{2}^{3} \\
t_{3}^{1} & t_{3}^{2} & t_{3}^{3}
\end{array}\right], \quad \mathbf{A}=\left[\begin{array}{ccc}
0 & \kappa_{3} & -\kappa_{2} \\
-\kappa_{3} & 0 & \kappa_{1} \\
\kappa_{2} & -\kappa_{1} & 0
\end{array}\right]
$$

and $\mathbf{Y}_{\mathbf{0}}$ containing the initial coordinates of the frame $\left(\mathbf{t}_{\mathbf{1}}, \mathbf{t}_{\mathbf{2}}, \mathbf{t}_{\mathbf{3}}\right)$ at one of the extremity. Equation (66) will be solved with the iterative method called Local Coordinates Approach [17. The iteration relies on the Magnus expansion which provides an exponential formulation of the solution of the first order matrix differential equation [29]. The interval of resolution is $\left[s_{n}, s_{n+1}\right]$ with $h=s_{n+1}-s_{n}$ the step of the method. The matrix $\mathbf{Y}_{\mathbf{n}}$ is the approximation of $\mathbf{Y}\left(s_{n}\right)$. Truncation of the Magnus expansion and approximation by the midpoint rule provides an iterative step of order 2 :

$$
\mathbf{Y}_{\mathbf{n}+\mathbf{1}}=\exp \left(h \mathbf{A}\left(s_{n}+\frac{h}{2}\right)\right) \mathbf{Y}_{\mathbf{n}}
$$

Equation (68) contains a matrix exponential. Let define the isomorphism $: \mathbb{R}^{3} \rightarrow \mathfrak{s o}(3)$ such that for $\boldsymbol{\theta} \in \mathbb{R}^{3}$ :

$$
\forall \mathbf{h} \in \mathbb{R}^{3}, \quad \hat{\boldsymbol{\theta}} \mathbf{h}=\boldsymbol{\theta} \times \mathbf{h}
$$


with:

$$
\boldsymbol{\theta}=\left[\begin{array}{c}
\theta_{1} \\
\theta_{2} \\
\theta_{3}
\end{array}\right], \quad \hat{\boldsymbol{\theta}}=\left[\begin{array}{ccc}
0 & -\theta_{3} & \theta_{2} \\
\theta_{3} & 0 & -\theta_{1} \\
-\theta_{2} & \theta_{1} & 0
\end{array}\right]
$$

and according to Simo et al. we have [4]:

$$
\exp (\hat{\boldsymbol{\theta}})=\cos (\|\boldsymbol{\theta}\|) \mathbf{I}+\sin (\|\boldsymbol{\theta}\|) \hat{\mathbf{e}}+(1-\cos (\|\boldsymbol{\theta}\|)) \mathbf{e} \otimes \mathbf{e}
$$

with $\mathbf{e}=\boldsymbol{\theta} /\|\boldsymbol{\theta}\|$. By posing:

$$
\boldsymbol{\lambda}=-h\left[\begin{array}{c}
\kappa_{1}\left(s_{n}+\frac{h}{2}\right) \\
\kappa_{2}\left(s_{n}+\frac{h}{2}\right) \\
\kappa_{3}\left(s_{n}+\frac{h}{2}\right)
\end{array}\right]
$$

we then have:

$$
\hat{\boldsymbol{\lambda}}=h \mathbf{A}\left(s_{n}+\frac{h}{2}\right)
$$

and using (71) we then have:

$$
\begin{aligned}
\exp \left(h \mathbf{A}\left(s_{n}+\frac{h}{2}\right)\right)= & \cos (\|\boldsymbol{\lambda}\|) \mathbf{I}+\sin (\|\boldsymbol{\lambda}\|) \hat{\boldsymbol{\mu}} \\
& +(1-\cos (\|\boldsymbol{\lambda}\|)) \boldsymbol{\mu} \otimes \boldsymbol{\mu}
\end{aligned}
$$

with $\boldsymbol{\mu}=\boldsymbol{\lambda} /\|\boldsymbol{\lambda}\|$. Consequently, the set $\left(\mathbf{Y}_{n}\right)_{n=0, . ., N}$ is obtained by iteration. Let $\gamma_{\boldsymbol{n}}$ be the following vector:

$$
\gamma_{\boldsymbol{n}}=\left[\begin{array}{c}
\gamma_{1}\left(s_{n}\right) \\
\gamma_{2}\left(s_{n}\right) \\
1+\gamma_{3}\left(s_{n}\right)
\end{array}\right]
$$

According to (11), the set $\left(\mathbf{x}_{\mathbf{1}_{n}}\right)_{n=0, . ., N}$ containing the approximations of the tangent to the beam neutral axis $\mathbf{x}_{\mathbf{0}}{ }^{\prime}\left(s_{i}\right)$ can be expressed by:

$$
\forall n \in \llbracket 0, N \rrbracket, \mathbf{x}_{\mathbf{1}_{n}}=\mathbf{Y}_{\mathbf{n}}^{T} \gamma_{\boldsymbol{n}}
$$

Let $\left(\mathbf{x}_{\mathbf{0}_{n}}\right)_{n=0, . ., N}$ be the set of points of the neutral axis of the beam for the discretization $\left(s_{n}\right)_{n=0, . ., N}$ with $\mathbf{x}_{\mathbf{0} 0}$ the coordinates of the beam extremity. This set can be calculated iteratively using the tangents obtained previously:

$$
\mathbf{x}_{\mathbf{0} n+1}=\mathbf{x}_{\mathbf{0} n}+h \mathbf{x}_{\mathbf{1} n}
$$

Finally, the deformed shape of the beam neutral axis has been reconstructed from strain measures. 


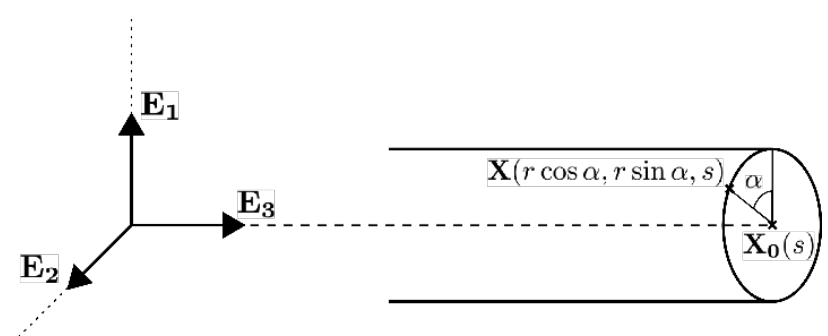

(a) Circular beam surface reference configuration

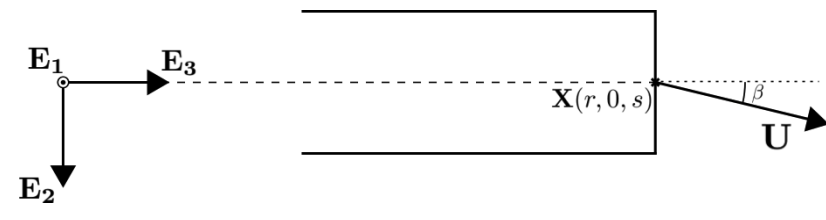

(b) Circular beam angle of orientation of the strain measure

Figure 4: Circular beam

\section{Beam shape sensing including bending, torsion and Poisson deformations : Application to circular beams}

This section proposes a method for beam shape sensing with a circular beam as an application of the theory presented in the previous section. As there is no warping for a beam with a circular cross section [4, the choice of this cross section geometry is consistent with the choice to not consider the out-of-plane deformation in the beam model. This application illustrates also the the possibility to select in the model the deformations to be taken into account. Here the bending and torsion deformation will be retrieve from the strain measures, to be used in the reconstruction, whereas the tensile and shearing deformation will not be exploited. This selection allows to decrease the number of strain measures to four instead of six without degrading the reconstruction accuracy as the tensile and shearing deformation have very little impact on it. They can thus be neglected compared to bending and torsion deformation. Consequently, only bending and torsion are taken into account in the strain configuration estimation and in the shape reconstruction. There is no impact on the estimation of bending and torsion as tensile and shearing are set together in the bias parameter which feature the unexploited parameters together. This choice meets the need to have a good cost-accuracy trade-off and the possibility, already developed in the litterature, to instrument a beam with four strain sensors on the cross sections $43,48,28,19,21$.

\subsection{System expression}

Let's consider a straight beam with circular cross section of radius $r$. Let $\boldsymbol{\xi}$ be a position at the surface of the beam parameterized with cylindrical coordinates, as illustrated in Fig. 4a.

$$
\boldsymbol{\xi}=\xi_{1} \mathbf{E}_{\mathbf{1}}+\xi_{2} \mathbf{E}_{\mathbf{2}}
$$

with

$$
\xi_{1}=r \cos \alpha, \quad \xi_{2}=r \sin \alpha, \quad \alpha \in[0,2 \pi[
$$

Let $\mathbf{U}$ be the vector tangent to the surface of the beam at location $\boldsymbol{\xi}$ and which makes an angle $\beta$ with $\mathbf{E}_{\mathbf{3}}$, 


$$
\begin{aligned}
& \mathbf{A}\left(\alpha_{k}, \beta_{k}, \nu\right)= \\
& {\left[\begin{array}{llll}
\left(\cos ^{2} \beta_{1}-\nu \sin ^{2} \beta_{1}\right) \sin \alpha_{1} & -\left(\cos ^{2} \beta_{1}-\nu \sin ^{2} \beta_{1}\right) \cos \alpha_{1} & \cos \beta_{1} \sin \beta_{1} & 1 \\
\left(\cos ^{2} \beta_{2}-\nu \sin ^{2} \beta_{2}\right) \sin \alpha_{2} & -\left(\cos ^{2} \beta_{2}-\nu \sin ^{2} \beta_{2}\right) \cos \alpha_{2} & \cos \beta_{2} \sin \beta_{2} & 1 \\
\left(\cos ^{2} \beta_{3}-\nu \sin ^{2} \beta_{3}\right) \sin \alpha_{3} & -\left(\cos ^{2} \beta_{3}-\nu \sin ^{2} \beta_{3}\right) \cos \alpha_{3} & \cos \beta_{3} \sin \beta_{3} & 1 \\
\left(\cos ^{2} \beta_{4}-\nu \sin ^{2} \beta_{4}\right) \sin \alpha_{4} & -\left(\cos ^{2} \beta_{4}-\nu \sin ^{2} \beta_{4}\right) \cos \alpha_{4} & \cos \beta_{4} \sin \beta_{4} & 1
\end{array}\right]}
\end{aligned}
$$

as shown in Fig. $4 \mathrm{~b}$, The angle $\beta$ will be the angle of orientation of the strain measure. The coordinates of $\mathbf{U}$ in the basis $\left(\mathbf{E}_{\mathbf{1}}, \mathbf{E}_{\mathbf{2}}, \mathbf{E}_{\mathbf{3}}\right)$ are:

$$
\mathbf{U}=\left[\begin{array}{c}
-\sin \alpha \sin \beta \\
\cos \alpha \sin \beta \\
\cos \beta
\end{array}\right]
$$

Based on 46 the strain $\varepsilon$ is now expressed as the scalar product between the beam position and direction vector $\mathbf{V} \in \mathbb{R}^{4}$ and the beam configuration vector $\mathbf{K} \in \mathbb{R}^{4}$ :

$$
\varepsilon=\mathbf{V}(\alpha, \beta, \nu) \cdot \mathbf{K}(r, \alpha, \beta, s, \nu)
$$

with

$$
\begin{aligned}
& V_{1}(\alpha, \beta, \nu)=\left(\cos ^{2} \beta-\nu \sin ^{2} \beta\right) \sin \alpha \\
& V_{2}(\alpha, \beta, \nu)=-\left(\cos ^{2} \beta-\nu \sin ^{2} \beta\right) \cos \alpha \\
& V_{3}(\alpha, \beta, \nu)=\cos \beta \sin \beta \\
& V_{4}(\alpha, \beta, \nu)=1
\end{aligned}
$$

and

$$
\begin{aligned}
& K_{1}(r, \alpha, \beta, s, \nu)=r \kappa_{1}(s) \\
& K_{2}(r, \alpha, \beta, s, \nu)=r \kappa_{2}(s) \\
& K_{3}(r, \alpha, \beta, s, \nu)=r \kappa_{3}(s) \\
& K_{4}(r, \alpha, \beta, s, \nu)=\delta(\alpha, \beta, s, \nu)
\end{aligned}
$$

with:

$$
\begin{aligned}
\delta(\alpha, \beta, s, \nu)= & -\sin \alpha \cos \beta \sin \beta \gamma_{1}(s) \\
& +\cos \alpha \cos \beta \sin \beta \gamma_{2}(s) \\
& +\left(\cos ^{2} \beta-\nu \sin ^{2} \beta\right) \gamma_{3}(s)
\end{aligned}
$$


The beam configuration vector $\mathbf{K}$ is composed of the bending parameters $\kappa_{1}$ and $\kappa_{2}$, the torsion paramereter $\kappa_{3}$ and $\delta$ containing the shearing and tensile parameters $\gamma_{1}, \gamma_{2}$ and $\gamma_{3}$. For a beam instrumented with 4 strain sensors on a cross section, with $\alpha_{i}$ and $\beta_{i}$ being the radial and axial angle of the $i^{\text {th }}$ sensor, we then have the following system:

$$
\mathbf{A}\left(\alpha_{k}, \beta_{k}, \nu\right) \mathbf{K}(r, \alpha, \beta, s, \nu)=\mathbf{B}
$$

The vector $\mathbf{B}$ contains the strain measures, with $B_{i}=\varepsilon_{i}$ and the expression of the matrix $\mathbf{A}$ is presented in (83). If $\beta_{1}=\beta_{2}=\beta_{3}=\beta_{4}$ the matrix $\mathbf{A}$ is not invertible because its third and fourth column are collinear, as shown in (83). Consequently, in order to solve the system (96) $\beta_{i}$ angles cannot be constant. The values of $\beta_{i}$ are set to the following values: $\beta_{1}=\beta, \beta_{2}=-\beta, \beta_{3}=\beta, \beta_{4}=-\beta$ with $\left.\beta \in\right] 0, \pi / 2\left[\right.$. The values of $\alpha_{i}$ are spaced with the same angle $\pi / 2$ as present in litterature [43, 48, 28, 19, 21]: $\alpha_{1}=0, \alpha_{2}=\frac{\pi}{2}, \alpha_{3}=\pi, \alpha_{4}=\frac{3 \pi}{2}$. The matrix $\mathbf{A}$ has then the following expression:

$$
\begin{aligned}
& \mathbf{A}\left(\alpha_{k}, \beta_{k}, \nu\right)= \\
& {\left[\begin{array}{ccrr}
0 & -\left(\cos ^{2} \beta-\nu \sin ^{2} \beta\right) & \cos \beta \sin \beta & 1 \\
\left(\cos ^{2} \beta-\nu \sin ^{2} \beta\right) & 0 & -\cos \beta \sin \beta & 1 \\
0 & \left(\cos ^{2} \beta-\nu \sin ^{2} \beta\right) & \cos \beta \sin \beta & 1 \\
-\left(\cos ^{2} \beta-\nu \sin ^{2} \beta\right) & 0 & -\cos \beta \sin \beta & 1
\end{array}\right]}
\end{aligned}
$$

It should be noted that for the value $\beta=\beta_{b}$ with $\beta_{b}=\tan ^{-1}(1 / \sqrt{\nu})$, the matrix $\mathbf{A}$ is not inversible. The reason can be explained by the value of $V_{1}$ and $V_{2}$ in 85 and 86 which are null for this specific value of $\beta_{b}$. In this case the strain $\varepsilon$ expressed in (84) only depends of torsion and shearing deformation. The strain measure $\varepsilon$ is then blind to bending and tensile deformation as this value of $\beta_{b}$ is the angle for which the axial strain due to these phenomenon and the transverse strain caused by the Poisson effect due to these phenomenon are cancelling each other. The angle $\beta$ is thus chosen such that $\beta \neq \beta_{b}$ and the expression of the inverse $\mathbf{A}^{-1}$ is then:

$$
\begin{aligned}
& \mathbf{A}^{-1}\left(\alpha_{k}, \beta_{k}, \nu\right)= \\
& \frac{1}{4}\left[\begin{array}{cccc}
0 & \frac{2}{\cos ^{2} \beta-\nu \sin ^{2} \beta} & 0 & \frac{-2}{\cos ^{2} \beta-\nu \sin ^{2} \beta} \\
\frac{-2}{\cos ^{2} \beta-\nu \sin ^{2} \beta} & 0 & \frac{2}{\cos ^{2} \beta-\nu \sin ^{2} \beta} & 0 \\
\frac{1}{\cos \beta \sin \beta} & \frac{-1}{\cos \beta \sin \beta} & \frac{1}{\cos \beta \sin \beta} & \frac{-1}{\cos \beta \sin \beta} \\
1 & 1 & 1 & 1
\end{array}\right]
\end{aligned}
$$

\subsection{Impact of Poisson effect}

The absence of the variable $\nu$ in the third row of the expression of $\mathbf{A}^{-\mathbf{1}}$ in 98 demonstrates that the Poisson effect does not have any influence on the estimation of the torsion parameter $\kappa_{3}$. In fact, as the torsion of a beam being the rotation of the cross sections between each other, the axial strain thus does not 


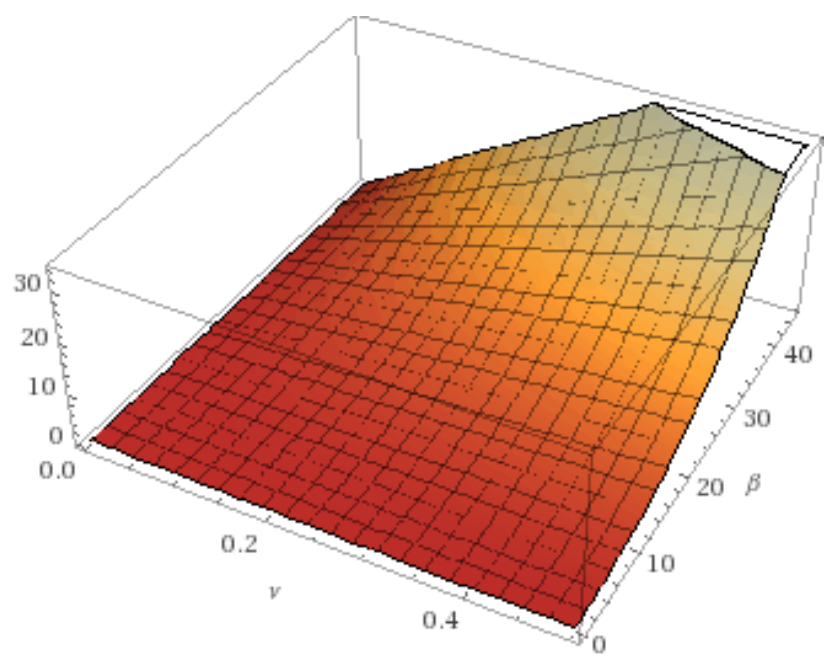

Figure 5: Impact of the non-inclusion of the Poisson effect on the estimates of the bending parameters $\kappa_{1}$ and $\kappa_{2}$ : the relative error of curvature estimation in percent is expressed according to to Poisson ratio $\nu$ and strain sensor orientation angle $\beta$.

have any impact on it. On the opposite, the presence of the variable $\nu$ in the first and second rows illustrates that the Poisson effect has an impact on the estimate of the bending parameters $\kappa_{1}$ and $\kappa_{2}$ :

$$
\begin{aligned}
& \kappa_{1}=\frac{\varepsilon_{2}-\varepsilon_{4}}{2 r\left(\cos ^{2} \beta-\nu \sin ^{2} \beta\right)} \\
& \kappa_{2}=\frac{-\varepsilon_{1}+\varepsilon_{3}}{2 r\left(\cos ^{2} \beta-\nu \sin ^{2} \beta\right)}
\end{aligned}
$$

The strain parameters estimated without taking into account the Poisson effect are noted $\kappa_{1}^{*}$ and $\kappa_{2}^{*}$ and are obtained from 99 and 100 by setting $\nu=0$. These parameters $\kappa_{i}^{*}$ can then be expressed in terms of $\kappa_{i}$ :

$$
\kappa_{i}^{*}=\left(1-\nu \tan ^{2} \beta\right) \kappa_{i}
$$

Finally, when the Poisson effect is not taken into consideration the estimate of the bending parameters are multiplied by the coefficient $\left(1-\nu \tan ^{2} \beta\right)$. Therefore, when the strain sensors measure the axial strain and are placed parallel to the beam $(\beta=0)$ these estimates are the same. This explains why works in the litterature with beam shape sensing methods where axial strains are considered produce good results without taking into account Poisson effect. The relative error of the estimates for the current beam instrumentation when the Poisson effect is not considered is illustrated in Fig. 5.

\section{Beam shape sensing including bending, torsion and Poisson deformation : Validation for circular beams}

This section presents the results of validation by simulation of the beam shape sensing method presented in Section 4 . The beam considered is a circular beam with a cross section diameter of $4 \mathrm{~mm}$ and a length 


\begin{tabular}{|c|c|c|c|c|}
\hline \multirow{2}{*}{ Deformation } & \multicolumn{4}{|c|}{ Loads } \\
\cline { 2 - 5 } & Type & Value & Location & Direction \\
\hline 1 & $\mathrm{~F}$ & $-10 \mathrm{~N}$ & $400 \mathrm{~mm}$ & Ox \\
\hline \multirow{3}{*}{2} & $\mathrm{~F}$ & $5 \mathrm{~N}$ & $200 \mathrm{~mm}$ & Ox \\
\cline { 2 - 5 } & $\mathrm{F}$ & $-10 \mathrm{~N}$ & $400 \mathrm{~mm}$ & Oy \\
\hline \multirow{3}{*}{3} & $\mathrm{~F}$ & $-10 \mathrm{~N}$ & $100 \mathrm{~mm}$ & Oy \\
\cline { 2 - 5 } & $\mathrm{M}$ & $-1000 \mathrm{~N} \cdot \mathrm{mm}$ & $200 \mathrm{~mm}$ & Oz \\
\cline { 2 - 5 } & $\mathrm{F}$ & $10 \mathrm{~N}$ & $300 \mathrm{~mm}$ & Ox \\
\cline { 2 - 5 } & $\mathrm{M}$ & $1000 \mathrm{~N} \cdot \mathrm{mm}$ & $400 \mathrm{~mm}$ & Oz \\
\hline \multirow{3}{*}{4} & $\mathrm{M}$ & $-1000 \mathrm{~N} \cdot \mathrm{mm}$ & $200 \mathrm{~mm}$ & Oz \\
\cline { 2 - 5 } & $\mathrm{F}$ & $-10 \mathrm{~N}$ & $400 \mathrm{~mm}$ & Oy \\
\hline \multirow{3}{*}{5} & $\mathrm{M}$ & $-200 \mathrm{~N} . \mathrm{mm}$ & $400 \mathrm{~mm}$ & Oz \\
\cline { 2 - 5 } & $\mathrm{F}$ & $-4 \mathrm{~N}$ & $400 \mathrm{~mm}$ & Oy \\
\hline \multirow{2}{*}{6} & $\mathrm{M}$ & $-1000 \mathrm{~N} \cdot \mathrm{mm}$ & $400 \mathrm{~mm}$ & Oz \\
\cline { 2 - 5 } & $\mathrm{F}$ & $1 \mathrm{~N}$ & $400 \mathrm{~mm}$ & Oy \\
\hline
\end{tabular}

Table 1: Load cases used for the simulations of beam deformation. The letter $\mathrm{F}$ and $\mathrm{M}$ denotes respectively a force and a moment.

of $400 \mathrm{~mm}$. The material of the beam is stainless steel and has a Poisson ratio of $\nu=0.30$. The initial geometry of the beam is straight, clamped at one of its extremity and free at its other. Different cases of deformations of beams subject to the static loads were simulated with FEM using ANSYS software. The method presented in Section 4 integrate in a full manner not only the bending but also the torsion deformations and how the strain due to that torsion is impacted by the Poisson effect. It is thus interesting to evaluate the reconstruction method accuracy on torsion deformations cases to assess the validity of the Poisson effect inclusion in the beam shape sensing method. In order to do so, the beam shape sensing method proposed in (4) is compared to beam shape sensing taking into account only bending deformation. The beam deformations considered are six cases of deformations with different intensity of torsion deformations for the purpose of sweeping out cases of very different mechanical loadings. The different loads applied in each of the cases are described in Table 1. Deformation 1 is a case of simple bending with a single load applied at the extremity of the beam. Deformation 2 is a case of combined bending with two loads applied orthogonally to each other at the middle and at the extremity of the beam. Deformation 3 is a case of bending combined with torsion, with two loads applied at $25 \%$ and $75 \%$ of the beam and two opposite moments of torsion applied at the middle and at the end of the beam. Deformation 4 is a case of bending combined with torsion, with a single load applied at the middle on the beam and a moment of torsion applied at the end 
of the beam. Deformation 5 and 6 are bending combined with torsion, with single load and moment of torsion applied at the end of the beam. Exemple of the deformed beam shapes are shown in Fig. 6a and Fig. 6b and their tip deflections, obtained from the deformed beam configurations of the simulation results, are presented in Table 2. Simulation results obtained with FEM are used to obtain the strain measures at

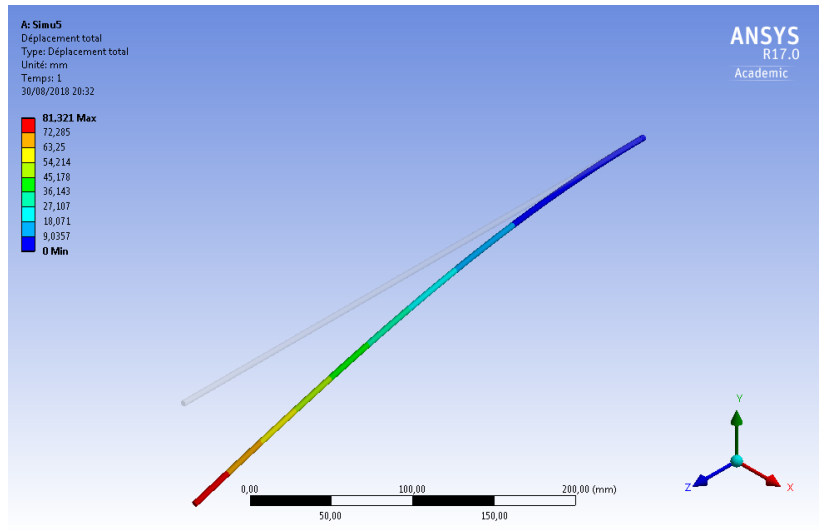

(a) Deformation 1: One force

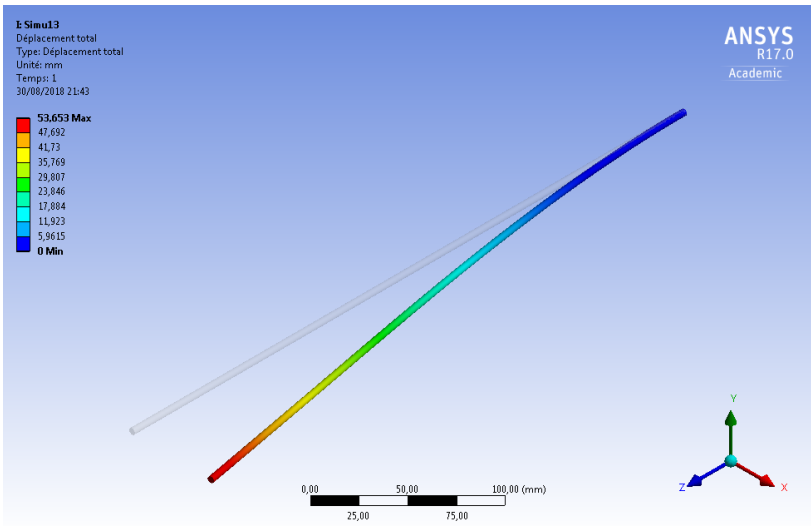

(b) Deformation 3: Two moments \& two forces

Figure 6: Beam deformations

the surface of the beam for any angle of orientation $\beta$. Using these strain measures as input data of beam shape sensing method allows to obtain the reconstructed beam shape. It is then possible to compare it with the actual deformed shape of the beam in order to evaluate the reconstruction accuracy of the beam shape method. The beam shape method proposed in Section 4, using 4 strain measures per intrumented cross sections will be used with the angle of strain measure $\beta=45^{\circ}$ to maximize the shear strain due to torsion. This method will be compared with a beam shape method derived also from Section 3 but using only 3 strain measures of axial strain $\left(\beta=0^{\circ}\right)$, as proposed in the litterature for circular beams [31, 33, 32, 18, 30, 2, 34, Tip distances between reconstructed shapes are used to assess of the quality of the reconstruction methods proposed. The reconstructed beam shapes are presented along with the actual beam shape in Fig. 7 for each of the six cases. The results of reconstruction tip errors are presented in Table 2, The beam length was discretized using a step of 2 millimeters. The reconstruction accuracy for deformation 1 is the same as both of the methods exhibit a tip error of $1.7 \mathrm{~mm}$. In case of deformation 2 and 3 the beam shape is better reconstructed with our method with tip errors of $1.7 \mathrm{~mm}$ and $1.6 \mathrm{~mm}$ compared to $1.9 \mathrm{~mm}$ and $2.0 \mathrm{~mm}$ with axial strain beam shape sensing method. Beam shapes reconstructions are exhibited in Fig. 7b and Fig. 7c. This improvement corresponds to a relative gain in accuracy of $11 \%$ and $20 \%$. Concerning deformations 4,5 and 6 the reconstructions are significantly better with our method, as shown in Fig. 7d, Fig. 7e and Fig. 7f. The reconstructed tip errors are thus $2.3 \mathrm{~mm}, 0.1 \mathrm{~mm}$ and $0.1 \mathrm{~mm}$ with our method compared to $7.5 \mathrm{~mm}$, $0.9 \mathrm{~mm}$ and $1.1 \mathrm{~mm}$ with beam shape sensing method using axial strain. This increase of reconstruction 
accuracy constitutes a relative gain of $70 \%, 89 \%$ and $91 \%$. Not only the absolute reconstruction tip errors are smaller with our method, but the relative tip errors are also more consistent as their values are not higher than $3 \%$ of the beam deflection, as opposed to beam shape sensing method using axial strain which presents a $9.1 \%$ and $12.9 \%$ relative tip error for deformation 4 and 6 according to Table 2 .

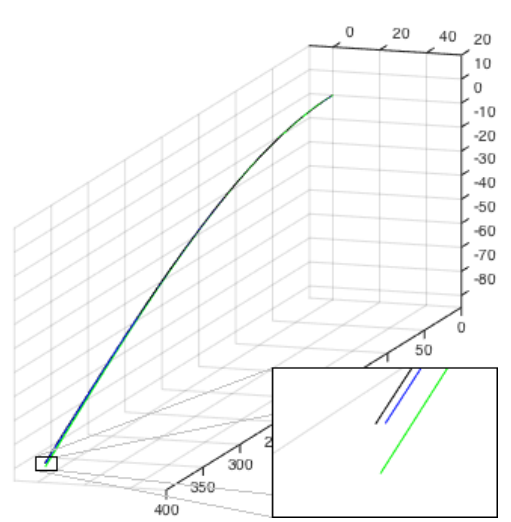

(a) Deformation 1

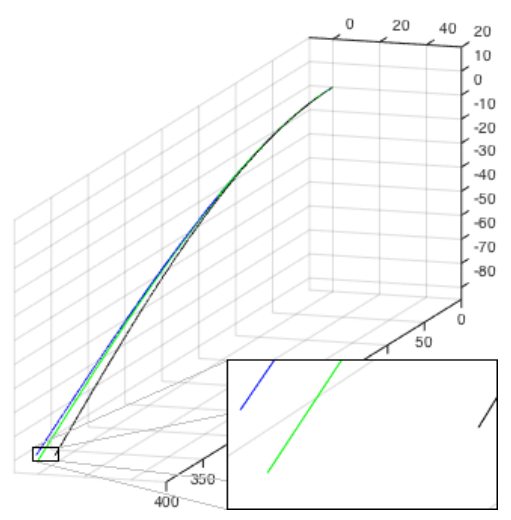

(d) Deformation 4

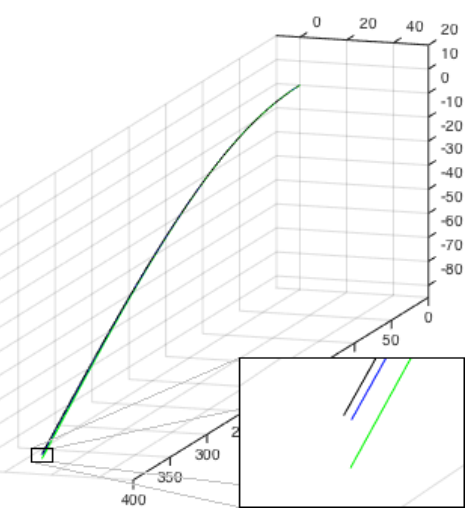

(b) Deformation 2

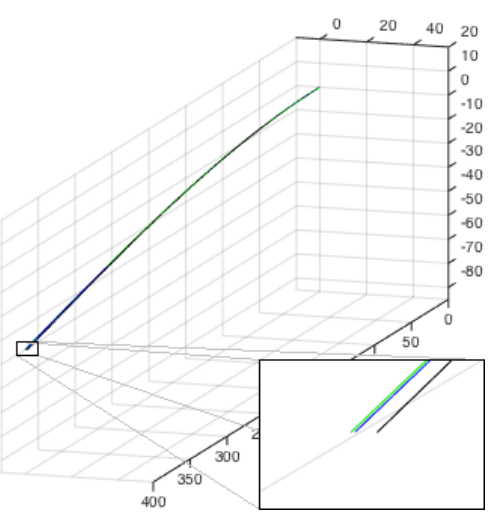

(e) Deformation 5

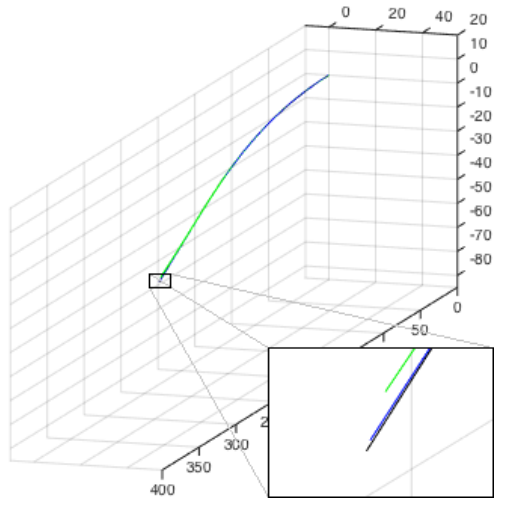

(c) Deformation 3

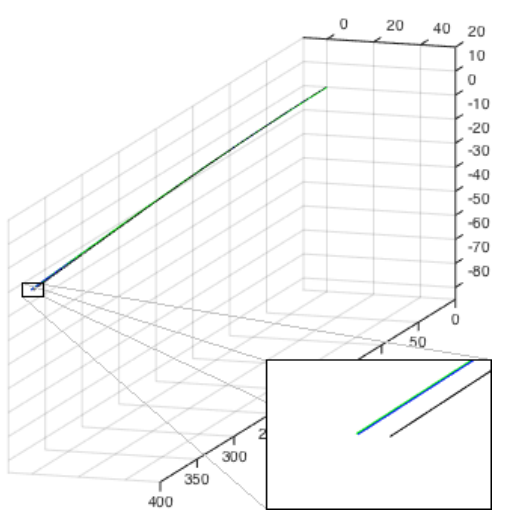

(f) Deformation 6

Figure 7: Original and reconstructed beam deformed shapes. The original beam shapes are represented in green. The beam shapes in blue have been reconstructed using 4 strain measures $\left(\beta=45^{\circ}\right)$ per cross sections and the beam shapes in black have been reconstructed using 3 axial strain measures $\left(\beta=0^{\circ}\right)$ per cross sections.

\section{Discussion}

In this article was presented a large deflection beam shape sensing method fully including the Poisson effect. In fact, the transversal strain caused by axial strain due to Poisson effect can have a significant impact on the beam strain. The Poisson effect has also an influence on the configuration of the cross section, but opposed to its influence on strains, the influence of the Poisson effect on sensor positions is minimal. The Poisson effect was thus included in an extended version of the small strain tensor and not in the beam space 


\begin{tabular}{|c|c|c|c|c|c|c|c|}
\hline \multirow{2}{*}{ Deformation } & \multirow{2}{*}{$\begin{array}{c}\text { Deflection } \\
\text { at free end }\end{array}$} & & \multicolumn{4}{|c|}{ Reconstruction tip error } & \multicolumn{2}{c|}{ Gain in accuracy } \\
\cline { 3 - 7 } & & Absolute & $\begin{array}{c}\text { Relative } \\
\text { of deflection) }\end{array}$ & Absolute & $\begin{array}{c}\text { Relative } \\
\text { of deflection) }\end{array}$ & \multirow{2}{*}{ Absolute } & Relative \\
\hline 1 & $81.3 \mathrm{~mm}$ & $1.7 \mathrm{~mm}$ & $2.1 \%$ & $1.7 \mathrm{~mm}$ & $2.1 \%$ & $0.0 \mathrm{~mm}$ & $0 \%$ \\
\hline 2 & $82.2 \mathrm{~mm}$ & $1.9 \mathrm{~mm}$ & $2.3 \%$ & $1.7 \mathrm{~mm}$ & $2.1 \%$ & $0.2 \mathrm{~mm}$ & $11 \%$ \\
\hline 3 & $53.4 \mathrm{~mm}$ & $2.0 \mathrm{~mm}$ & $3.7 \%$ & $1.6 \mathrm{~mm}$ & $3.0 \%$ & $0.4 \mathrm{~mm}$ & $20 \%$ \\
\hline 4 & $82.0 \mathrm{~mm}$ & $7.5 \mathrm{~mm}$ & $9.1 \%$ & $2.3 \mathrm{~mm}$ & $2.8 \%$ & $5.2 \mathrm{~mm}$ & $70 \%$ \\
\hline 5 & $33.7 \mathrm{~mm}$ & $0.9 \mathrm{~mm}$ & $2.7 \%$ & $0.1 \mathrm{~mm}$ & $0.3 \%$ & $0.8 \mathrm{~mm}$ & $89 \%$ \\
\hline 6 & $8.5 \mathrm{~mm}$ & $1.1 \mathrm{~mm}$ & $12.9 \%$ & $0.1 \mathrm{~mm}$ & $1.2 \%$ & $1.0 \mathrm{~mm}$ & $91 \%$ \\
\hline
\end{tabular}

Table 2: Beam tip deflections of the deformed beams and reconstruction tip errors according to the sensors configurations.

configuration. This method was described previously in the litterature but to the author's knowledge has not been used before for beam shape sensing. A small-strain tensor including Poisson effect is thus obtained and can then be used to express the strain parameters in terms of strain measures. The large deflection property of the beam shape sensing method is preserved thanks to the objectivity of this tensor which is due to the geometrically-exact characteristics of the beam theory used. It results that the beam shape sensing is then able to handle bending, torsion, shearing and tensile-compression deformations.

A practical exemple of beam shape sensing was presented with a circular straight beam of $400 \mathrm{~mm}$ length and instrumented with 4 strain sensors per cross sections with an angle of orientation $\beta=45^{\circ}$. The number of 4 strains measures was dictated by the cost-accuracy trade-off governing real instrumentation. It was then chosen as a compromise between the necessity to handle deformations having the most impact on the shape reconstruction (bending and torsion) and the need to limit the number of strain measures by cross section. The goal of this restriction to bending and torsion is also to facilitate the comparison with other method only taking into account bending. The principal difference then residing in the inclusion of torsion, it is then possible to evaluate its impact on reconstruction from adequate cases of deformation. The reconstruction results can then be used to evaluate the accuracy of the beam shape sensing method proposed and specifically of the beam model with Poisson effect.

The deformations cases were conceived to contained various level of torsion. In every case flexion has been added by application of point loads because reconstruction of the beam neutral axis under torsion only would have been unnoticeable. The shear center is the point on the cross section of the beam where the application of loads does not cause its twisting. As the cross section of the beam is circular and thus symmetrical, the shear center of the beam is the same as the center of gravity and is thus the neutral axis. Deformation 1 is a case of simple bending and as the load is applied on the center of the cross section there 
is no torsion. Deformation 2 is a case of combined bending caused by two orthogonal loads, one at the center of the beam and the other at the extremity. Deformation 3 is a combination of bending and torsion by application of two loads and two opposed moments applied on the middle and the extremity of the beam. The last three deformations are combination of bending and torsion with a single moment of torsion applied in each case. It is suggested that these are the cases where the torsion intensity is the highest. In the case of deformation 4 the moment of torsion is applied at the center of the beam and is then null between the center and the free extremity. For deformations 5 and 6 the moment is applied at the extremity of the beam and is then uniform the whole length of the beam. The moment value is higher in case of deformation 6 .

Even though it can seem logical that the beam shape sensing methods with 4 strain measures per cross section produce better reconstruction results than the method with 3 strain measures, it should be noted that: 1) the gain in accuracy is significant, 2) the more the torsion is, the higher the gain is. Taking into account torsion thus allow to reconstruct more accurately some case of deformations. Here, deformation 1 does not include torsion and is thus reconstructed with the same accuracy by both methods. The deformation 2 and 3 have limited torsion (deformation 2 because the torsion is induced by the double bending and deformation 3 because the two moments applied are have opposed signs). The gain for those two reconstructions with our method is then limited. In the case of deformation 4, 5 and 6 the torsion is high due to the property of the loads: the gain of accuracy is thus significant with our method. Our method then give better or even results than the classical beam shape sensing methods using axial strains. The beam shape sensing method proposed thus take into account effectively torsional deformations. Finally, these results validate our approach concerning the Poisson effect and the beam shape sensing method proposed.

\section{Conclusion}

In this article a new large deflection beam shape method including Poisson effect was presented. Simulation results showed the improvement of reconstruction accuracy obtained with this method compared to beam shape method using axial strain in case of deformations not limited to bending. This validates the relevancy of the approach concerning the inclusion of the Poisson effect in the beam model and the beam shape sensing method proposed. Future works will include experimental beam shape reconstructions, optimal angle of strain measures, impact of sensor positioning accuracy on parameters estimations, beam load reconstruction from strain measures as well as addition of the out-of-plane warping effect.

\section{Acknowledgments}

This work is part of the project GAME-D, financed by the French National Agency for Research (ref: ANR-12- TECS-0019) and supported by Laboratory of Excellence CAMI (ref: ANR-11-LABX-0004-01). 


\section{Declaration of interests}

None

\section{References}

[1] Abayazid, M., Kemp, M., \& Misra, S. (2013). 3d flexible needle steering in soft-tissue phantoms using fiber bragg grating sensors. In Proc. IEEE International Conference on Robotics and Automation (ICRA) (pp. 5843-5849). doi 10.1109/ ICRA.2013.6631418

[2] Araujo, F. M., Ferreira, L. A., \& Santos, J. L. (2002). Simultaneous determination of curvature, plane of curvature, and temperature by use of a miniaturized sensing head based on fiber bragg gratings. Appl. Opt., 41, 2401-2407. URL: http://ao.osa.org/abstract.cfm?URI=ao-41-13-2401 doi 10.1364/A0.41.002401.

[3] Askins, C. G., Miller, G. A., \& Friebele, E. J. (2008). Bend and twist sensing in a multiple-core optical fiber. In OFC/NFOEC 2008 - 2008 Conference on Optical Fiber Communication/National Fiber Optic Engineers Conference (pp. 1-3). doi 10.1109/OFC.2008.4528404.

[4] Bauchau, O. A., \& Craig, J. I. (2009). Euler-bernoulli beam theory. In O. A. Bauchau, \& J. I. Craig (Eds.), Structural Analysis (pp. 173-221). Dordrecht: Springer Netherlands. URL: http://dx.doi.org/10.1007/978-90-481-2516-6_5 doi 10.1007/978-90-481-2516-6_5.

[5] Beck, A. T., \& da Silva, C. R. (2011). Timoshenko versus euler beam theory: Pitfalls of a deterministic approach. Structural Safety, 33, 19 - 25. URL: http://www.sciencedirect.com/science/article/pii/S0167473010000597 doi https://doi. org $/ 10.1016 / \mathrm{j}$. strusafe.2010.04.006

[6] Chadha, M., \& Todd, M. D. (2017). A generalized approach for reconstructing the three-dimensional shape of slender structures including the effects of curvature, shear, torsion, and elongation. Journal of Applied Mechanics, 84, 041003.

[7] Chadha, M., \& Todd, M. D. (2019). A comprehensive kinematic model of single-manifold cosserat beam structures with application to a finite strain measurement model for strain gauges. International Journal of Solids and Structures, 159, 58 - 76. URL: http://www.sciencedirect.com/science/article/pii/S0020768318303767. doi https://doi.org/ $10.1016 /$ j.ijsolstr.2018.09.020

[8] Chadha, M., \& Todd, M. D. (2019). An improved shape reconstruction methodology for long rod like structures using cosserat kinematics- including the poisson's effect. In G. Kerschen (Ed.), Nonlinear Dynamics, Volume 1 (pp. 237-246). Cham: Springer International Publishing.

[9] Chan, T. H. T., Ashebo, D. B., Tam, H., Yu, Y., Chan, T., Lee, P., \& Gracia, E. P. (2009). Vertical displacement measurements for bridges using optical fiber sensors and ccd cameras. Structural Health Monitoring, 8, 243-249. URL: https://doi.org/10.1177/1475921708102108 doi 10.1177/1475921708102108 arXiv:https://doi.org/10.1177/1475921708102108

[10] Cheng, B., Zhu, W., Liu, J., Yuan, L., \& Xiao, H. (2017). 3d beam shape estimation based on distributed coaxial cable interferometric sensor. Smart Materials and Structures, 26, 35-44. URL: http://stacks.iop.org/0964-1726/26/i=3/a= 035017.

[11] Chung, W., Kim, S., Kim, N.-S., \& up Lee, H. (2008). Deflection estimation of a full scale prestressed concrete girder using long-gauge fiber optic sensors. Construction and Building Materials, 22, 394 - 401. URL: http://www.sciencedirect. com/science/article/pii/S0950061806002443 doi https://doi.org/10.1016/j.conbuildmat.2006.08.007

[12] Crisfield, M. A., \& Jelenic;, G. (1999). Objectivity of strain measures in the geometrically exact three-dimensional beam theory and its finite-element implementation. Proceedings of the Royal Society of London. Series A: Mathematical, Physical and Engineering Sciences, 455, 1125-1147. URL: https://royalsocietypublishing.org/doi/abs/10.1098/rspa.1999. 0352 doi 10.1098/rspa.1999.0352 arXiv:https://royalsocietypublishing.org/doi/pdf/10.1098/rspa.1999.0352 
[13] Davis, M. A., Kersey, A. D., Sirkis, J., \& Friebele, E. J. (1996). Shape and vibration mode sensing using a fiber optic bragg grating array. Smart Materials and Structures, 5, 759. URL: http://stacks.iop.org/0964-1726/5/i=6/a=005

[14] Gherlone, M., Cerracchio, P., Mattone, M., Sciuva, M. D., \& Tessler, A. (2012). Shape sensing of 3d frame structures using an inverse finite element method. International Journal of Solids and Structures, 49, 3100 - 3112. URL: http://www. sciencedirect.com/science/article/pii/S0020768312002648 doi https://doi.org/10.1016/j.ijsolstr.2012.06.009

[15] Gherlone, M., Cerracchio, P., Mattone, M., Sciuva, M. D., \& Tessler, A. (2014). An inverse finite element method for beam shape sensing: theoretical framework and experimental validation. Smart Materials and Structures, 23, 45-57. URL: http://stacks.iop.org/0964-1726/23/i=4/a=045027

[16] Glaser, R., Caccese, V., \& Shahinpoor, M. (2012). Shape monitoring of a beam structure from measured strain or curvature. Experimental Mechanics, 52, 591-606. URL: https://doi.org/10.1007/s11340-011-9523-y doi 10.1007/ s11340-011-9523-y

[17] Hairer, E., Wanner, G., \& Lubich, C. (2006). Geometric Numerical Integration. Structure-Preserving Algorithms for Ordinary Differential Equations. (2nd ed.).

[18] Henken, K. R., Dankelman, J., van den Dobbelsteen, J. J., Cheng, L. K., \& van der Heiden, M. S. (2014). Error analysis of fbg-based shape sensors for medical needle tracking, . 19, 1523-1531. doi 10.1109/TMECH.2013.2287764

[19] Kang, H.-I. K. L.-H., \& Han, J.-H. (2009). Real-time structure shape estimation using distributed fiber bragg grating sensors. In Proceedings of the ASME Conference on Smart Materials, Adaptive Structures, and Intelligent Systems (p. 461). ASME.

[20] Kapania, R. K., \& Li, J. (2003). A formulation and implementation of geometrically exact curved beam elements incorporating finite strains and finite rotations. Computational Mechanics, 30, 444-459. URL: https://doi.org/10.1007/ s00466-003-0422-7, doi 10.1007/s00466-003-0422-7

[21] Kim, H.-I., Kang, L.-H., \& Han, J.-H. (2011). Shape estimation with distributed fiber bragg grating sensors for rotating structures. Smart Materials and Structures, 20, 035011. URL: http://stacks.iop.org/0964-1726/20/i=3/a=035011

[22] Kim, N. S., \& Cho, N. S. (2004). Estimating deflection of a simple beam model using fiber optic bragg-grating sensors. Experimental Mechanics, 44, 433-439. URL: https://doi.org/10.1007/BF02428097. doi 10.1007/BF02428097

[23] Kim, S.-W., Kang, W.-R., Jeong, M.-S., Lee, I., \& Kwon, I.-B. (2013). Deflection estimation of a wind turbine blade using fbg sensors embedded in the blade bonding line. Smart Materials and Structures, 22, 125004. URL: http://stacks.iop. org/0964-1726/22/i=12/a=125004

[24] Li, D.-K., \& Li, X.-F. (2016). Large deflection and rotation of timoshenko beams with frictional end supports under three-point bending. Comptes Rendus Mécanique, 344, 556-568. doi $10.1016 / j . c r m e .2016 .01 .007$

[25] Linn, J., Lang, H., \& Tuganov, A. (2013). Geometrically exact cosserat rods with kelvin-voigt type viscous damping. Mechanical Sciences, 4, 79-96. URL: https://www.mech-sci.net/4/79/2013/ doi 10.5194/ms-4-79-2013

[26] Liu, H., Farvardin, A., Pedram, S. A., Iordachita, I., Taylor, R. H., \& Armand, M. (2015). Large deflection shape sensing of a continuum manipulator for minimally-invasive surgery. In 2015 IEEE International Conference on Robotics and Automation (ICRA) (pp. 201-206). doi 10.1109/ICRA.2015.7139000.

[27] Liu, L., \& Lu, N. (2016). Variational formulations, instabilities and critical loadings of space curved beams. International Journal of Solids and Structures, 87, 48 - 60. URL: http://www.sciencedirect.com/science/article/pii/ S0020768316000949 doi https://doi.org/10.1016/j.ijsolstr.2016.02.032

[28] Lunwei, Z., Jinwu, Q., Linyong, S., \& Yanan, Z. (2004). Fbg sensor devices for spatial shape detection of intelligent colonoscope. In Robotics and Automation, 2004. Proceedings. ICRA '04. 2004 IEEE International Conference on (pp. 834-840 Vol.1). volume 1. doi 10.1109/ROBOT.2004.1307253

[29] Magnus, W. (1954). On the exponential solution of differential equations for a linear operator. Communications on Pure and Applied Mathematics, 7, 649-673. URL:http://dx.doi.org/10.1002/cpa.3160070404 doi 10.1002/cpa.3160070404 
[30] Moon, H., Jeong, J., Kang, S., Kim, K., Song, Y.-W., \& Kim, J. (2014). Fiber-bragg-grating-based ultrathin shape sensors displaying single-channel sweeping for minimally invasive surgery. Optics and Lasers in Engineering, 59, 50 - 55. doi http://dx.doi.org/10.1016/j.optlaseng.2014.03.005

[31] Moore, J. P., \& Rogge, M. D. (2012). Shape sensing using multi-core fiber optic cable and parametric curve solutions. Opt. Express, 20, 2967-2973. URL: http://www.opticsexpress.org/abstract.cfm?URI=oe-20-3-2967 doi 10.1364/OE. 20.002967

[32] Park, Y.-L., Elayaperumal, S., Daniel, B., Ryu, S. C., Shin, M., Savall, J., Black, R., Moslehi, B., \& Cutkosky, M. (2010). Real-time estimation of 3-d needle shape and deflection for mri-guided interventions, . 15, 906-915. doi 10.1109/TMECH. 2010.2080360

[33] Roesthuis, R., Kemp, M., van den Dobbelsteen, J., \& Misra, S. (2014). Three-dimensional needle shape reconstruction using an array of fiber bragg grating sensors, . 19, 1115-1126. doi 10.1109/TMECH.2013.2269836

[34] Roesthuis, R. J., \& Misra, S. (2016). Steering of multisegment continuum manipulators using rigid-link modeling and fbg-based shape sensing, . 32, 372-382. doi 10.1109/TR0.2016.2527047

[35] Saravia, C. M. (2014). A large deformation-small strain formulation for the mechanics of geometrically exact thin-walled composite beams. Thin-Walled Structures, 84, 443 - 451. URL: http://www.sciencedirect.com/science/article/pii/ S0263823114001724 doi https://doi.org/10.1016/j.tws.2014.05.014

[36] Schaefer, P.-L., Barrier, G., Chagnon, G., Alonso, T., \& Moreau-Gaudry, A. (2019). Strain gauges based 3d shape monitoring of beam structures using finite width gauge model. Experimental Techniques, . URL: https://doi.org/10. 1007/s40799-019-00312-4 doi 10.1007/s40799-019-00312-4.

[37] Schaefer, P.-L., Chagnon, G., \& Moreau-Gaudry, A. (2019). Optimized needle shape reconstruction using experimentally based strain sensors positioning. Medical \& Biological Engineering \& Computing, . doi 10.1007/s11517-019-02001-1

[38] Seifabadi, R., Gomez, E. E., Aalamifar, F., Fichtinger, G., \& Iordachita, I. (2013). Real-time tracking of a bevel-tip needle with varying insertion depth: Toward teleoperated mri-guided needle steering. In 2013 IEEE/RSJ International Conference on Intelligent Robots and Systems (pp. 469-476). doi 10.1109/IROS.2013.6696393

[39] Sigurdardottir, D. H., Stearns, J., \& Glisic, B. (2017). Error in the determination of the deformed shape of prismatic beams using the double integration of curvature. Smart Materials and Structures, 26. URL: http://stacks.iop.org/ $0964-1726 / 26 / i=7 / a=075002$

[40] Simo, J. (1985). A finite strain beam formulation. the three-dimensional dynamic problem. part i. Computer Methods in Applied Mechanics and Engineering, 49, 55 - 70. URL: http://www.sciencedirect.com/science/article/pii/ 0045782585900507, doi http://dx.doi.org/10.1016/0045-7825(85)90050-7

[41] Simo, J., \& Fox, D. (1989). On a stress resultant geometrically exact shell model. part i: Formulation and optimal parametrization. Computer Methods in Applied Mechanics and Engineering, 72, 267 - 304. doi http://dx.doi.org/10. 1016/0045-7825(89)90002-9

[42] Todd, M. D., Stull, C. J., \& Dickerson, M. (2013). A local material basis solution approach to reconstructing the threedimensional displacement of rod-like structures from strain measurements. Journal of Applied Mechanics, 80.

[43] Wang, H., Zhang, R., Chen, W., Liang, X., \& Pfeifer, R. (2016). Shape detection algorithm for soft manipulator based on fiber bragg gratings. IEEE/ASME Transactions on Mechatronics, 21, 2977-2982. doi 10.1109/TMECH.2016.2606491

[44] Weiss, H. (2002). Dynamics of geometrically nonlinear rods: I. mechanical models and equations of motion. Nonlinear Dynamics, 30, 357-381. URL: https://doi.org/10.1023/A:1021268325425 doi 10.1023/A:1021268325425

[45] Xu, H., Ren, W.-X., \& Wang, Z.-C. (2015). Deflection estimation of bending beam structures using fiber bragg grating strain sensors. Advances in Structural Engineering, 18, 395-403.

[46] Xu, R., Yurkewich, A., \& Patel, R. V. (2016). Shape sensing for torsionally compliant concentric-tube robots. URL: http://dx.doi.org/10.1117/12.2213128 doi 10.1117/12.2213128. 
[47] Yau, M. H., Chan, T., Thambiratnam, D. P., \& Tam, H. Y. (2013). Static vertical displacement measurement of bridges using fiber bragg grating (fbg) sensors. Advances in Structural Engineering, 16, 165-176. URL: https://doi.org/10. 1260/1369-4332.16.1.165 doi 10.1260/1369-4332.16.1.165 arXiv:https://doi.org/10.1260/1369-4332.16.1.165

[48] Yi, X., Qian, J., Shen, L., Zhang, Y., \& Zhang, Z. (2007). An innovative 3d colonoscope shape sensing sensor based on fbg sensor array. In Information Acquisition, 200\%. ICIA '07. International Conference on (pp. 227-232). doi 10.1109/ ICIA.2007.4295731

[49] Zhang, B., Chen, F., Yang, M., Huang, L., Du, Z., Sun, L., \& Dong, W. (2018). Real-time curvature detection of a flexible needle with a bevel tip. Sensors, 18, 2057.

[50] Zhao, Z., Soto, M. A., Tang, M., \& Thévenaz, L. (2016). Distributed shape sensing using brillouin scattering in multicore fibers. Opt. Express, 24, 25211-25223. URL: http://www.opticsexpress.org/abstract.cfm?URI=oe-24-22-25211 doi $10.1364 /$ EE.24.025211 